OPEN ACCESS

Edited by:

Eric O. Long,

National Institute of Allergy and Infectious Diseases,

United States

Reviewed by:

Emily Mace,

Baylor College of Medicine, United States

Amir Horowitz,

Icahn School of Medicine at Mount Sinai,

United States

*Correspondence:

Haiming Wei

ustcwhm@ustc.edu.cn

Specialty section:

This article was submitted to

NK and Innate Lymphoid

Cell Biology,

a section of the journal

Frontiers in Immunology

Received: 02 May 2017

Accepted: 22 August 2017 Published: 05 September 2017

Citation:

Zhou Y, Xu X, Tian Z and Wei H (2017) "Multi-Omics" Analyses of the Development and Function of Natural Killer Cells.

Front. Immunol. 8:1095. doi: 10.3389/fimmu.2017.01095

\section{"Multi-Omics" Analyses of the Development and Function of Natural Killer Cells}

\author{
Yonggang Zhou', Xiuxiu Xu ${ }^{1,2}$, Zhigang Tian ${ }^{1,2}$ and Haiming Wei, ${ }^{1,2 *}$ \\ 'School of Life Science and Medical Center, Institute of Immunology, CAS Key Laboratory of Innate Immunity and Chronic \\ Disease, University of Science and Technology of China, Hefei, China, ${ }^{2}$ Hefei National Laboratory for Physical Sciences at \\ Microscale, University of Science and Technology of China, Hefei, China
}

For over four decades, our understanding of natural killer (NK) cells has evolved from the original description of cluster of differentiation (CD) $56^{+}{ }^{+} \mathrm{CD}^{-}$to establishing NK cells as an important subset of innate lymphocytes in the host's surveillance against viral infections and malignancy. The progress of research on the fundamental properties and therapeutic prospects for translational medicine using NK cells excites immunologists and clinicians. Over the past decade, numerous advances in "-omics"-scale methods and new technological approaches have addressed many essential questions in the biology of NK cells. We now have further understanding of the overall molecular mechanisms of action that determine the development, function, plasticity, diversity, and immune reactivity of NK cells. These findings are summarized here, and our view on how to study NK cells using "multi-omics" is highlighted. We also describe "-omics" analyses of the relationships between NK cells and viral infection, tumorigenesis, and autoimmune diseases. Ultimately, a deeper and more comprehensive understanding of NK cells in multiple conditions will provide more effective strategies to manipulate NK cells for the treatment of human disease.

Keywords: "omics" technology, "multi-omics", natural killer cell molecular program, natural killer cell diversity, natural killer cell immunotherapy

\section{INTRODUCTION}

As early as 1975, in some experiments carried out in vitro, a phenomenon was noticed: some lymphocytes of an undefined type from the normal mouse spleen selectively fought against Moloney leukemia cells spontaneously (1). In 1979, the same cell functions were also described in healthy humans $(2,3)$. The phenomenon was described as "natural cytotoxicity," and the lymphocytes were ultimately named "natural killer" (NK) cells.

Initially, NK cells were believed to act as just an "annoying" background of cytolytic activity in several cell lineages. In 1986, as a result of the discovery of several cell surface markers and the confirmation of natural cytotoxicity, NK cells were determined to be a new lineage of lymphocytes $(4,5)$. The first 30 years of research into NK cells were focused mainly on descriptions of the functions and the identification of single surface markers. The overall progress into research of NK cells was slow and lagged behind that of most other types of immune cells.

Over the past decade, developments in "-omics"-scale technology, such as analyses of gene expression as well as quantification of proteins and metabolites, have enriched our understanding of the complex biologic processes of NK cells. This understanding includes their phylogeny, 
developmental programs, plasticity, and immune reactivity for controlling viral infections and malignancy at the molecular level. More importantly, NK cells have recently attracted attention for their therapeutic prospects in cellular immunotherapy due to technical progress that has helped immunologists and clinicians gain a better understanding of NK cells (6).

The English-language neologism "-omics" contains several specific molecular levels, such as "genomics" (the sequence and expression of DNA), "transcriptomics" (DNA transcription into RNA), "proteomics" (RNA translation into proteins), or "metabolomics" (metabolites). These methods generate large data sets, which are often referred to as "-omics" data. The expansion in "-omics" methods is due to mainly tremendous advancements in technology through approaches such as next-generation sequencing (NGS) and mass spectrometry (MS).

In this review, we highlight the "-omics"-scale data that have assisted research of NK cells (Figure 1), including methods that examine their phenotypes, transcriptional signatures, and effector functions in various biologic processes or niches. This approach can help to constantly update the "road map" of gene expression that forms a more comprehensive regulation network and provides new strategies to manipulate NK cells for the treatment of human disease.

\section{"OMICS" TECHNOLOGY APPLIED TO THE STUDY OF NK CELLS}

Aside from metabolomics, most “-omics” technologies are usually considered to be based on genomics, which arose largely from the deciphering of the complete human genome $(7,8)$ and mouse genome (9) in the early 2000s, undertakings that marked a new milestone in the life sciences. "Omics" technology presents a panoramic view of the unbiased molecular determinants of NK cells not only from development to an exhaustive process but also for multiple responses to effector function in different environments. To analyze NK cells using different levels of "-omics" approaches, very different biotechnologies are applied in each case.

\section{Microarrays Technology}

Due to the increasing efficiency of chips (10) and the constantly increasing number of available monoclonal antibodies, thousands of biologic reactions at DNA, RNA, or protein levels can be measured or even quantified (11) in a single experiment. As the earliest high-throughput method to analyze gene transcription and protein expression, microarray technology (12) (Figure 1) has made significant contributions to the rapid development of research of NK cells over the last decade. Although NK cells have been considered for a long time to be natural soldiers against viral infection and cancer in the body, the key transcription factors (TFs) that regulate the responses of NK cells to viral infection are poorly understood.

Therefore, to screen key TFs, researchers used microarray technology to compare and analyze changes in gene expression in purified $\mathrm{Ly}_{4} 9 \mathrm{H}^{+} \mathrm{NK}$ cells from murine cytomegalovirus (MCMV)-infected and control mice (13) (Table 1). In this experiment alone, $>30,000$ genes were evaluated on a microarray, and $Z b t b 32$ was screened because it was one of the most highly upregulated genes after MCMV infection. These data were confirmed through quantitative reverse transcription-polymerase

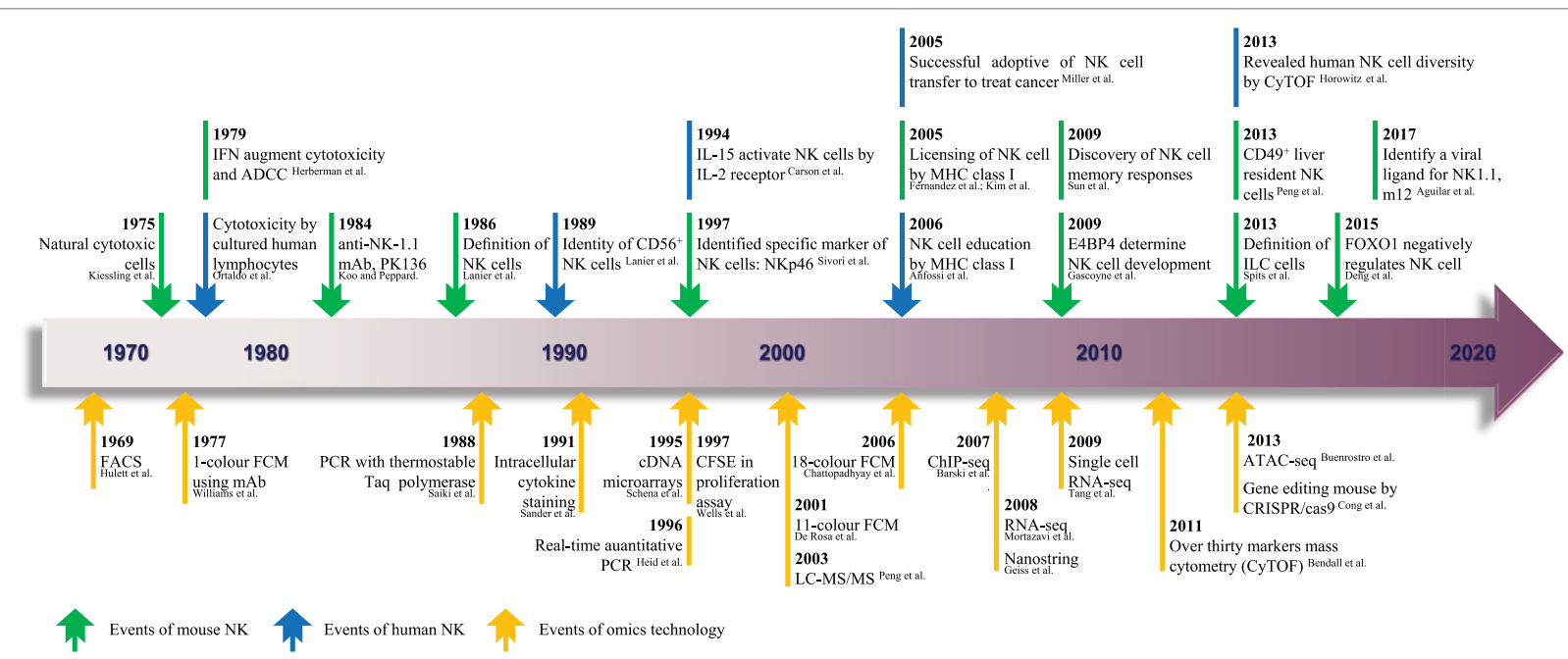

FIGURE 1 | The timeline of NK cell research keeps pace with advances in "-omics" technology. Since the identification of NK cells in 1975, analytical methods for NK cells were based mainly on FCM and gene expression analyses. From 1969 to the present day, rapid technological advances in FCM and gene expression analyses have become high-throughput technologies in the true sense, and there is much hope for the future. The top row shows the timeline of events related to NK cell research. Blue represents NK cells in general, and green denotes mouse NK cells. The bottom row shows the progress of events related to "-omics" technology. NK, natural killer; ADCC, antibody-dependent cell-mediated cytotoxicity; mAb, monoclonal antibody; ILC, innate lymphoid cell; FACS, fluorescenceactivated cell sorting; FCM, flow cytometry; PCR, polymerase chain reaction; CFSE, carboxyfluorescein succinimidyl ester; ChIP-seq, chromatin immunoprecipitation sequencing; RNA-seq, RNA sequencing; ATAC-seq, assay for transposase-accessible chromatin sequencing; CRISPR, clustered regularly interspaced short palindromic repeats; LC-MS/MS, liquid chromatography-tandem mass spectrometry. The references cited in this figure are all listed in the data sheet (Supplementary Material). 
TABLE 1 | Application of "Omics technologies" in complex NK cell research.

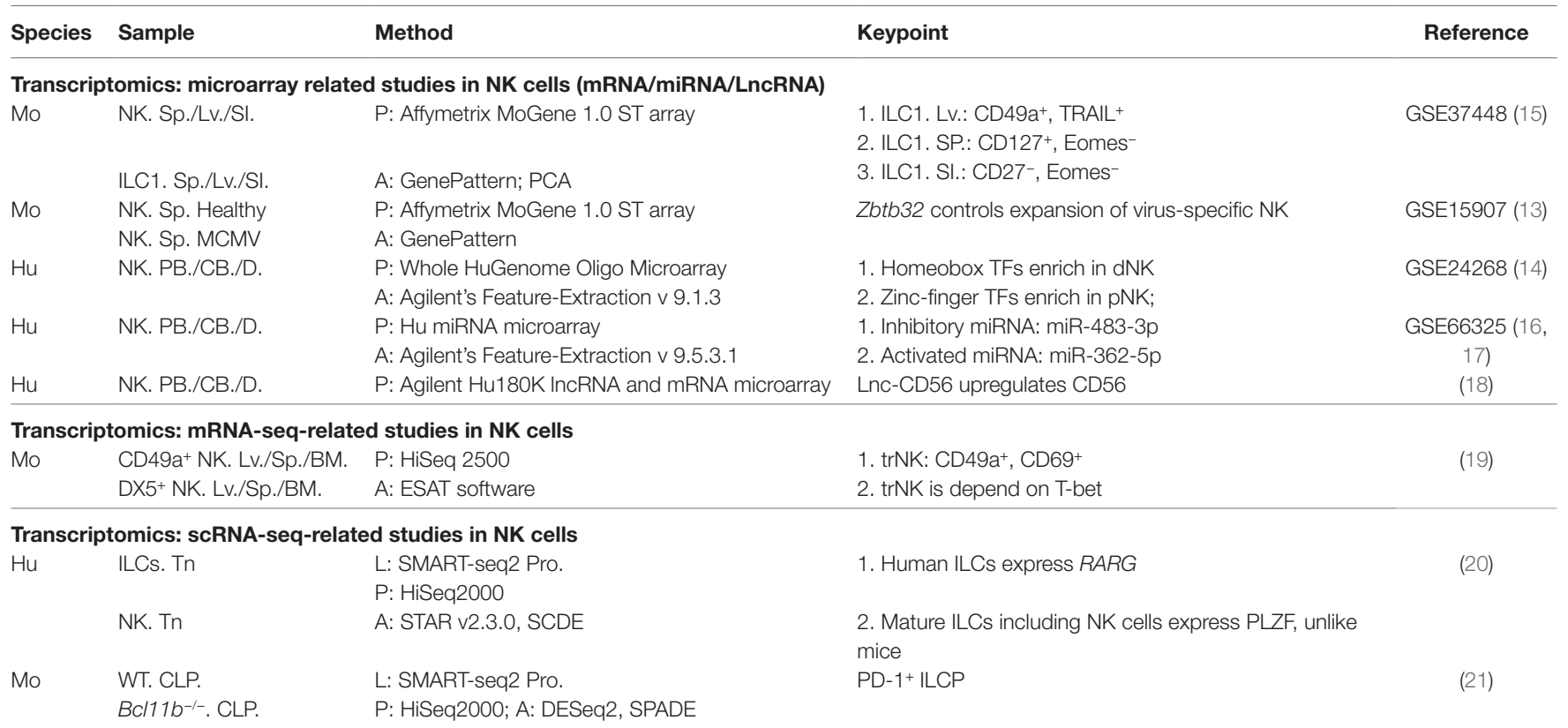

Transcriptomics: miRNA-seq-related studies in NK cells

Mo NK. Sp. Resting P: GA (lllumina) seq; SOLiD seq

NK. Sp. IL-15-activated A: pipeline $v$ 0.2.2, SHRiMP

Transcriptomics: ATAC-Seq related studies in NK cells

$\begin{array}{lll}\text { NK. Sp./LV. } & \text { ATAC-Seq, P: HiSeq } 2500 & \text { Regulomes of ILCS VS T cells: } \\ \text { ILC1. LV. } & \text { A: MACS v 1.4.2, HOMER v 4.8 } & \\ \text { HSC. BM. } & \text { ChIP-Seq, P: HiSeq 2500 } & \text { 1. The regulator of the ILC effector genes is easier to } \\ \text { CLP. BM. } & \text { A: SICER, MACS V } 1.4 .2 & \text { open } \\ \text { NKp. BM. } & \text { RNA-seq, P: HiSeq } 2000 & \text { 2. Regulomes of ILCs arborize early at precursor stages }\end{array}$

imNK. BM. A: Cufflinks 2.2.1

Proteomic: CyTOF-related studies in NK cells

$\begin{array}{lll}\mathrm{Hu} & \text { NK. CB./PB. Healthy } & \text { P: Mass cytometer (Fluidigm) }\end{array}$

(24)

Hu NK. PB. $\quad$ P: Mass cytometer (Fluidigm)

NK. L-PXF. A: SPADE; Cytobank

CD49e- trNK in human liver

(25)

Proteomic: LC-MS/MS-related studies in hematopoietic cells (focused on NK cells)

The effect genes of NK and $\mathrm{T}_{\mathrm{EM}}$ cells are similar

CD56 ${ }^{\text {dim }}$ NK. PB. A: MaxQuant v1.5.3.2, Communication

PXD004352 (26)

CRISPR-related studies in NK cells

Mo SFRs ${ }^{-/-} \mathrm{NK}$.

P: CRISPR

SFRs for NK cell education

(27)

A: Sequencing; FACS

Mo, mouse; Hu, Human; Sp, spleen; Lv, Liver; BM, bone marrow; SI, small intestinal lamina propria; L-PxF, liver postexcision flush; PB, peripheral blood mononuclear cell; CB, cord blood mononuclear cells; D, decidual mononuclear cells; Tn, tonsil; SFRs, signaling lymphocytic activation molecule family receptors library preparation; TFs, transcription factors; HSCs, hemopoietic stem cells; CLP, common lymphoid progenitors; trNK, tissue-resident natural killer; NKp, natural killer cell precursor; imNK, immature natural killer; CNK, conventional natural killer; ILCP, precursors of innate lymphoid cells; L, library preparation; P, platform; A, analysis; Pro., protocol; scRNA-seq, single-cell RNA sequencing; ATAC-Seq, assay for transposase-accessible chromatin sequencing; ChIP-seq, chromatin immunoprecipitation sequencing; CyTOF, cytometry by time of flight; LC-MS/MS, liquid chromatography-tandem mass spectrometry; CRISPR, clustered regularly interspaced short palindromic repeats; SCDE, single-cell differential expression; SPADE, spanning-tree progression analysis of density-normalized events; SICER, Spatial clustering for identification of ChIP-enriched regions; UHPLC, ultra-high performance liquid chromatography; CD, cluster of differentiation; dNK, decidual natural killer; PNK, peripheral natural killer; RNA-seq, RNA sequencing; ILC, innate lymphoid cell; IL, interleukin; ncRNA, non-coding RNA; FACS, fluorescence-activated cell sorting; HIV, human immunodeficiency virus; MCMV, murine cytomegalovirus.

chain reaction. This experiment is a classic instance of how to screen key genes in an important biologic process by microarray analysis. In addition, microarray technology is also used widely for studying the phenotypic and functional molecular signatures of NK cells. Wang and colleagues, using sorted populations of human NK cells from decidual, cord blood, and peripheral blood, investigated novel phenotypic and functional molecular signatures and transcriptional regulators by whole-genome microarray analysis (14) (Table 1). Through a comparative analysis of gene profiles of NK cells from those sources, the authors highlighted the differences in surface receptors, chemokine receptors, TFs, and functional molecules of NK cell populations. Interestingly, that research indicated that decidual natural killer $(\mathrm{dNK})$ cells may specifically express some new 
growth factors, cytokines, and chemokine genes; the identification of these genes is helpful for the functional classification of dNK cells. More notably, they showed that TF expression in $\mathrm{dNK}$ cells and peripheral natural killer ( $\mathrm{pNK}$ ) cells has family preferences: dNK cells are enriched for the homeobox family, whereas pNK cells express zinc-finger family TFs predominantly. The two studies mentioned above have been cited extensively by other researchers in cell biology.

Based on microarray technology, immunologists and computational biologists proposed the Immunological Genome Project (ImmGen), which is currently building a gene expression database for all characterized immune cells in the mouse (28). All data generated as a part of ImmGen are available freely and publicly on www.immgen.org.

\section{RNA Sequencing (RNA-Seq)}

At the height of use of microarray technology, researchers intending to study gene profiles used gene arrays. However, in 2005, Solexa technology (Illumina) and SOLiD technology (Life Technologies) emerged as key symbols in the evolution of NGS. As the cost of sequencing plummeted, RNA-seq became an increasingly popular method of transcriptome analysis. Unlike microarray technology (which relies on fluorescent labeling), RNA-seq mainly transforms RNA into a cDNA library, which is followed by direct sequencing (29) (Figure 1). Under the condition of sufficient sequencing depth, RNA-seq is applied to analyze the differential elements of gene expression of the whole transcriptome in a more accurate, reproducible, wider, and more reliable manner than that of other methods $(30,31)$.

In addition to analyzing the levels of gene expression, RNAseq can also identify new transcripts and splice variants and can measure allele-specific gene expression. Therefore, RNA-seq applied to analyses of the gene expression profile in these areas has more advantages than that of microarray analysis. RNAseq has many advantages, but several researchers continue to use chips, especially if the sample size is large. Because its data processing is fast and simple and the raw RNA data are troublesome, bioinformatists are required to adopt different strategies of data analysis based on the design and target of the experiment. Currently, some RNA-seq data analysis programs have been published and some professional analytical software has been updated constantly.

RNA sequencing has also been applied in the research of NK cells. Since cluster of differentiation (CD) $49 a^{+} \mathrm{DX}^{-}$was identified as the iconic marker of tissue-resident natural killer (trNK) cells in the liver (32), research on trNK cells has moved rapidly. To characterize the molecular profile of trNK cells in the liver more precisely, RNA-seq was used to analyze puri-

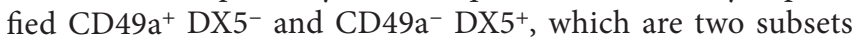
of NK cells from the liver, spleen, and bone marrow of mice (19) (Table 1). Results showed that trNK cells in the liver are a unique lineage of mature NK cells that are different from several reported NK cell subsets (19). By contrast, a basic hierarchical clustering analysis among different populations revealed that liver trNK cells displayed DX5 ${ }^{-} \mathrm{CD} 49 \mathrm{a}^{+} \mathrm{CD} 69^{+} \mathrm{CD} 44^{+} \mathrm{CD} 160^{+}-$ specific signatures and were depend on T-box expressed in $\mathrm{T}$ cells (T-bet) and not nuclear factor, interleukin 3 regulated
(NFIL3) (19) (Table 1). Wang et al. and Sojka et al. showed that microarray and RNA-seq technologies were useful to analyze the differences between NK cell subsets and for a comprehensive assessment of new subsets. However, better performance, lower costs, and help from bioinformatics have led RNA-seq to be favored. Furthermore, RNA-seq technology is also improving to help solve more complex problems.

\section{Single-Cell RNA Sequencing (scRNA-Seq)}

There is now a general consensus that cell heterogeneity is common and normal. Whether microarray or RNA-seq technology need to extract a bulk RNA from more than $10^{5}$ cells, and the data obtained are the average values of cell populations (33). These methods cannot meet the demand of immunologists to study the diversity of immune cells, and even some important information may be ignored.

Recently, with technological advances in the separation of single cells and the establishment of cDNA libraries, scRNAseq technology has emerged to make it easier to analyze the molecular profile of the single cell from cell populations (34) (Figure 1). Innate lymphoid cells (ILCs), including helper-like cells (ILC1, ILC2, and ILC3) and conventional natural killer (cNK) cells (35), are a new paradigm of immune cells that mirror the helper $\mathrm{T}$ cell subsets that produce similar functional molecules $(36,37)$. ScRNA-seq as an important technological advance of RNA-seq that can provide great opportunities for ILCs research. As a highly heterogeneous cell population and with a restriction of cell numbers, studying the developmental trajectory and signatures of ILC progenitor cells is a problem. To address this problem, researchers delineated distinct ILC development stages and reported that PD- $1^{\text {hi }}$ could be used as a marker of ILC precursor cells by undertaking scRNA-seq of bone marrow progenitor cells (21) (Table 1). The identification of PD- $1^{\text {hi }}$ ILC precursor cells had a positive effect on tumor immunotherapy of PD-1 antibody (21). As research has progressed, scholars have now identified a brand new level of complexity in biology.

\section{MicroRNA Sequencing (miRNA-Seq)}

miRNA molecules, although small, are powerful regulators of gene expression, and they are also expected to be markers of the diagnosis and therapeutic targets of a particular disease. Most of the research on the miRNA of NK cells can be done through microarray technology. This approach has led to the discovery of the inhibitory miRNA miR-483-3p (16) and the activated miRNA miR-362-5p (17) in human NK cells (Table 1).

However, if microarray technology and miRNA-seq are compared, the latter may have some obvious advantages. miRNA-seq can overcome the limitations of microarray technology (which is reliant on known miRNAs) to identify new miRNAs. miRNA-seq can even detect the difference in a single base of miRNAs. To improve the detection resolution and screen new miRNAs that regulate the function of NK cells, miRNA-seq was used to analyze the changes of miRNAs across the whole transcriptome during the activation of NK cells in mouse spleens by interleukin (IL)-15 (22) (Table 1). The library of this project was completed through two sequencing platforms: GA (Illumina) and SOLiD. Although 
there were small differences between the results of the two sequencing platforms, some new miRNAs were identified, and miR-223 was found to be an important regulator that inhibited the activation of NK cells (22).

\section{Assay for Transposase-Accessible Chromatin Sequencing (ATAC-Seq)}

Similar to miRNAs, TFs are important elements of gene expression. However, TF functions are dependent on the specific and accessible chromatin regions in the genome. Currently, the most common methods used for the identification of accessible chromatin regions are chromatin immunoprecipitation sequencing (ChIP-seq) (38) and ATAC-seq (39) (Figure 1).

Chromatin immunoprecipitation sequencing can directly detect DNA sequences that bind to TFs, but a single sequencing can only provide information about a definitive TF (38). ATAC-seq requires only a few cells and simple experimental steps, and, after sequencing, all the accessible chromatin regions of chromosomes at a particular time and space can be obtained, and these are not confined to a TF-binding site or a specific area of histone acetylation (40). As mentioned above, the study of the transcriptional regulatory elements within a cell is essential for a comprehensive understanding of how the cell operates. To conduct a panoramic study on the transcriptional regulatory elements of ILCs, Shih and colleagues used ATAC-seq to analyze all of the prototypical subsets of ILCs from mice, including cNK cells (23) (Table 1). Focusing on the regulatory elements of the functional genes of ILCs, they showed that ILCs and T cells expressed similar functional genes to resist infection, but that the gene-regulatory elements of ILCs were more likely to be activated (23).

\section{Mass Cytometry}

Compared with genes, proteins are the main components of life activities. Thus, biologists have been eager to implement highthroughput detection of cellular proteins. Flow cytometry (FCM) based on antibodies coupled with fluorescent compounds is the most common method used to analyze the proteins expressed on cells (41). To avoid overlap between wavelengths, the number of samples researchers can process is limited, so this method cannot achieve high-resolution detection.

Recently, a novel technology termed mass cytometry also known as cytometry with time-of-flight mass spectrometry (CyTOF) (42) (Figure 1) was developed. This merging of FCM and MS was developed to provide measurements of $>40$ coinstantaneous cellular parameters at single-cell resolution, and it has enhanced the detection ability considerably to explore complicated cellular systems (43). CyTOF allows for single-cell analysis of a larger number of markers than conventional FCM. A study by Bendall and colleagues demonstrated the applications of this technology for the first time using hematopoiesis, and the data were analyzed by spanning-tree progression analysis of density-normalized events (SPADE) (42). Then, the technology was demonstrated by applying the use of human leukocyte antigen (HLA) class-I tetramers to identify and model antigenspecific T cells (44).

By using CyTOF to analyze the expression of human pNK cell receptors in five sets of monozygotic twins, Horowitz and Blish described an unexpected scale of NK cell diversity and provided valuable evidence for an unsubstantiated hypothesis that genetic factors can control the expression of inhibitory receptors, whereas environmental factors may alter the expression of activated receptors $(45,46)$ (Table 1 ). Whereafter, by using CyTOF to study CMV reactivation in transplantation settings for acute myeloid leukemia (AML), Horowitz and colleagues discovered strong associations with HLA-C upregulation and increased expression of inhibitory killer cell immunoglobulinlike receptor (KIR) on effector memory CD8 T cells (47). CyTOF has also been used to measure cytokine-induced memory-like NK cells that were expected to be used in AML therapy by Todd Fehniger's group (48). Another study by Blish and colleagues on NK cell diversity associated with antiviral function made good use of CyTOF (24).

Although there have been some reports of MS being used for immunologic studies, conventional FCM based on fluorescence continues to dominate and maintain a valuable role in the immunologist's toolbox. This problem could be because (i) MS is limited by slow detection speed so a large number of samples cannot be detected; (ii) unique requirements for antibody labeling lead to the price of an individual panel being higher; and (iii) a method to sort and purify the detected cell population of interest is not available. Nevertheless, we believe that the continuous improvement and wider application of CyTOF will provide more useful data to immunologists with regard to complex subsets of immune cells.

\section{Liquid Chromatography-Tandem Mass Spectrometry (LC-MS/MS)}

Cytometry by time of flight can detect the expression of $>30$ proteins in a single sample, but the extremely complex proteomics of cells cannot be evaluated. Nevertheless, a panoramic image of the cell proteome is needed urgently (26).

LC-MS/MS (i.e., a LC separator combined with a tandem MS detector) is a versatile, highly accurate, highly sensitive, and automated method for the qualitative and quantitative analyses of most small molecules. LC-MS/MS was first used for the study of yeast proteomics in 2003 (49) (Figure 1). Rieckmann and colleagues demonstrated a new, complex, and comprehensive research project of 28 hematopoietic cell types by single-shot LC-MS/MS (26) (http://www.immprot.org/). By recording the differences, clustering, and principal component analysis of different cells, they showed that, based on the relationship between functional proteins, a complex "social network" can be formed among immune cells and that the nearest partner of NK cells are $\mathrm{CD}^{+} \mathrm{T}$ effector memory cells (26) (Table 1). These findings are similar to the results of ATAC-seq described above, and both sets of findings were reported using big data analyses to show that NK cells have the same antiinfection function as adaptive immune cells.

\section{Gene Knockout with Clustered Regularly Interspaced Short Palindromic Repeats (CRISPR)}

Immunologists are keen to confirm the functions of genes or proteins. Gene knockout mice have long been considered the gold 
standard for functional analyses in vivo. CRISPR/Cas9 is a newly developed gene-editing technology $(50,51)$ (Figure 1). CRISPR/ Cas9 is very exciting because it not only greatly improves the efficiency of gene knockout in mice $(52,53)$ but also makes it possible to construct mice with simultaneous knockouts in multiple genes. Chen and colleagues used CRISPR/Cas9 genome-editing technology, and, through the distribution of multi-point gene targeting, they knocked-out 10 genes in signaling lymphocytic activation molecule (SLAM) receptors and SLAM-associated protein family proteins in mice. These animals revealed a new mechanism of acquisition of NK cell function and solved the issue of "SLAM family receptor redundancy," which has been recognized as a problem in this research field (27) (Table 1). Biologists around the world are riding a wave of new technologies made possible by CRISPR.

From qPCR to microarray, from RNA-seq to scRNA-seq, and from traditional FCM to MS, “-omics" technologies are undergoing rapid development and will continue to be updated and enriched. These "-omics" technologies are facilitating a revolution in the research of the developmental and functional analyses of immune cells. The massive amounts of data generated using these methods are critical for understanding the contributions of immune cells to disease prevention and also for taking advantage of their full potential in immune cell-based therapies.

\section{"OMICS" TECHNOLOGIES POWER FURTHERING UNDERSTANDING OF NK CELLS}

Thanks to the tireless efforts of immunologists, the study of NK cells has made great progress, and we have a more extensive understanding of NK cells. However, due to their complexity, three major research questions regarding NK cells remain: (i) understanding of NK cells from multiple perspectives (what is a NK cell?); (ii) the origin and development of NK cells (where do NK cells come from?); and whether NK cells can be transformed and applied (where are NK cells going?) (Figure 2).
These problems are complicated, but they can be divided into different levels and solved using a single "-omics" or a combination of multiple "-omics" ("multi-omics") (Figure 2). "Omics" analyses are based on selecting the "-omics" technology and making the corresponding programs according to the experimental target.

First, in the study of the characteristics of NK cells, molecular profiles (e.g., transcriptome, proteome) were often analyzed comparatively among different cell types (e.g., NK cell subsets, NK cells, and ILCs) by microarray (14), RNA-seq (19), and CyTOF (25) (Table 1). Those studies revealed the unique profiles of gene expression or protein expression of different types of NK cells, but did not detail the key molecular mechanisms or carry out integration of data analyses to identify new regulatory elements. Furthermore, the "multi-omics" analysis of RNA-seq and mass spectrometric can also greatly improve the reliability of data and compensate for the shortage of data repeatability of small samples. But in terms of the project by Rieckmann and colleagues, it contains only the most classic human NK cell subsets: CD56 $6^{\text {bright }}$ and CD56 ${ }^{\text {dim }}$, although it is the credible and ambitious resource (26). In addition, the diversity of NK cell receptors has been a problem for researchers (54), especially the KIR (human) or Ly49 (mouse) families. "Multi-omics" could be used to study their regulomes by combining ATAC-seq with RNA-seq (23).

Second, innate immunity is a protective mechanism present in many types in plants and animals and even in prokaryotes $(5,55)$. The phylogeny of NK cells is not well understood. NK-like cells and some important receptor families related to the receptors of NK cells in mammals have been confirmed in bony fishes, amphibians, reptiles, and birds (56-58). In addition, with the rapid development of NGS technology and after mapping of the human and mouse genomes, a great deal of species-level genome sequencing has been completed. These big data sets of genomics could help decipher the phylogeny of NK cells according to the cluster of characteristic genes that contain multiple aspects of NK cells.

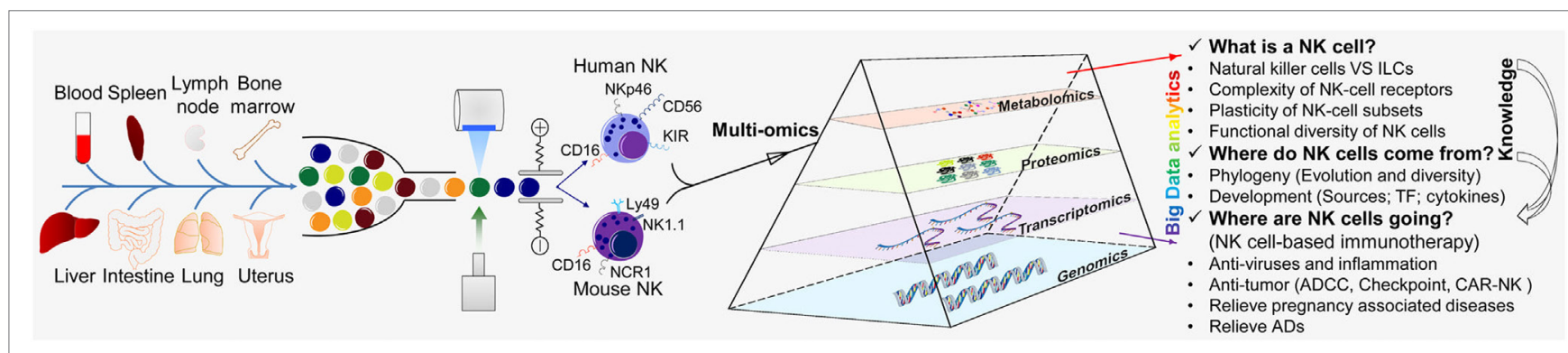

FIGURE 2 | "Multi-omics" analyses for the further understanding of NK cells. "Multi-omics" analytical design and key questions to be addressed for NK cells (schematic). NK cells are an important part of the first line of defense for the body. NK cells are present in most of the tissues and organs of humans and mice, including blood, spleen, lymph nodes, bone marrow, liver, lungs, and uterus. NK cells were isolated by flow cytometry and analyzed by various "-omics" technologies in steady and activated states. Just like a prism refracting the seven colors comprising white light, "-omics" and "multi-omics" analyses of the intricate critical problems of NK cells can produce massive amounts of data and a panoramic view by sequencing, mass spectrometry, and LC-MS/MS and could help to solve these problems. There are three major research aspects of NK cells: (i) understanding NK cells from multiple perspectives ("what is a NK cell?"); (ii) the origin and development of NK cells ("where do NK cells come from?"); and (iii) how NK cells may be transformed and applied ("where are NK cells going?"). The knowledge provided by basic research can guide and serve the clinical transformation of NK cells. TF, transcription factor; ADCC, antibody-dependent cell-mediated cytotoxicity; CAR-NK, chimeric antigen receptor-engineered natural killer cell; NK, natural killer cell; ILC, innate lymphoid cell. 
Third, in the study of disease-related NK cells, "multi-omics" that contain transcriptome, proteome, and even metabolome is an effective means of research (Figure 2). Paired single-cell analyses by scRNA-seq, RNA-seq, and CyTOF in combination have been used to describe the immune environment in lung cancer tissues and showed that the number of NK cells is severely reduced and impaired during the progression of lung cancer (59). It has been suggested that tumor immunotherapy of NK cells may be effective only in the early stage of lung cancer, but a new therapeutic target or possible methods are lacking.

\section{DISSECTING THE WHOLE TRANSCRIPTOME NETWORK OF NK CELL DEVELOPMENT}

From NFIL3, the first relatively specific TF $(60,61)$ reported, to Forkhead box protein O1 (FOXO1), the first negative TF (62) reported, the past decade has seen a sharp increase in research of the transcriptional regulation of NK cell development.

Nuclear factor, interleukin 3 regulated is a crucial regulator for the early development of NK cells and commitment to the NK lineage because $\mathrm{Nil3}^{-/}$mice exhibit impaired production of NK cells at the transition of NK precursor cells to immature NK cells in the bone marrow (60). NFIL3 acts in the positive feedback loop of the IL-15 receptor (CD122) (63) by determining the expression of the downstream TFs Id 2 and eomesodermin (EOMES) directly $(60,64,65)$. Although several TFs have roles in NK cell development, not only Eomes but also T-bet regulate the development and function of NK cells (66). T-bet is known to be the critical TF of interferon (IFN)- $\gamma$ production downstream of the IL-12 pathway and drives the development of T-helper 1 cells (67). With regarding to NK cells in the bone marrow, $T b \times 21^{-/-}$mice can block the production of NK cells at the transition from stage III $\left(\mathrm{CD} 27^{+} \mathrm{CD} 11 \mathrm{~b}^{+}\right)$to stage IV $\left(\mathrm{CD} 27^{-} \mathrm{CD} 11 \mathrm{~b}^{+}\right)(68)$. Many target genes of T-bet and EOMES necessary for the appropriate development of NK cells and selective regulation of effector functions have been identified, such as Ifn- $\gamma$, Granzyme B, Perforin, Blimp1, and S1p5 (68-70). T-bet and EOMES synergize the transcriptional regulation of cytotoxic factors in NK cells (66). Because T-bet is so important, several recent studies have focused on the negative factors or checkpoints for T-bet. FOXO1 downregulates T-bet expression (62) or mothers against decapentaplegic homolog 3 (SMAD3) downregulates NFIL3 expression (71) to impair the maturation and function of NK cells. Although those studies have used various "-omics" technologies and gene knockout mice, they have not described the entire transcriptional regulatory network of NK cell development due to a lack of research on posttranscriptional regulation.

It is also becoming evident that the development and functions of NK cells are not only regulated by TFs but are also influenced by posttranscriptional regulation through noncoding RNAs (ncRNAs) (72). Recent studies have shown that ncRNAs, including miRNAs, that are short ncRNAs (19-26 nt) and long ncRNAs (>200 nt), are also important for the development and function of NK cells $(73,74)$. Microarray analyses have been used to screen miRNAs in different NK cells from different tissues and shown that miR-483-3p decreases the cytotoxicity of NK cells due to inhibition of activated signal transducer and activator of transcription 5 by insulin-like growth factor 1 (16). Studies have also shown that miR-362-5p facilitates the function of NK cells by downregulating deubiquitinating enzyme CYLD expression (17). A similar experimental approach was used to analyze long ncRNA differences in NK cells from different tissues, and a novel long ncRNA, lnc-CD56, was identified, which positively regulates CD56 in human NK cells (18).

Although some progress has been made, research in this area is relatively scarce. More importantly, the transcriptional regulation program of NK cell development is a "cat's cradle" of networks performing at multiple levels. Thus, as with any single-factor analysis, understanding the molecular program of NK cell development completely is challenging. "Multi-omics" can help (i) predict and analyze new regulatory elements and (ii) better understand the molecular mechanisms of transcriptional regulation in NK cell development (Figure 2).

\section{“OMICS" ANALYSIS SHEDS LIGHT ON THE DIVERSITY OF NK CELLS}

Traditionally, NK cells have been thought to be a homogenous population derived from the bone marrow and which circulate throughout peripheral tissues. In recent years, studies have shown that NK cells constitute various unique subsets with different phenotypes and functions $(45,75,76)$.

From the perspective of NK cells in the liver, in 2013, Tian's group is the first to identify CD $49 \mathrm{a}^{+} \mathrm{DX} 5^{-} \mathrm{NK}$ cells as trNK cells. Through a comprehensive transcriptome obtained via microarray and fluorescence-activated cell sorting analyses $(32,77-80)$, they suggested that lineages of trNK cells in the liver may be different from $\mathrm{cNK}$ cells in the spleen. The discovery of liver trNK cells has rejuvenated scholars and will lead to trNK research in other areas (81). In 2014, Yokoyama's group showed that CD49a ${ }^{+}$trNK cells are present not only in the liver but also in the skin and uterine tissue (19). RNA-seq and multiple TF gene deficiencies in mice were used to provide more complete evidence to answer why trNK cells are different to cNK cells, especially in terms of TFs requirements. Their data confirmed the notion that the development of trNK cells in the liver is independent of GATA-3 or NFIL3 but dependent on T-bet (19). After that discovery, it was revealed that a "T-bet ${ }^{+}$Eomes $^{-}$CD $49 \mathrm{a}^{+} \mathrm{NK}$ cell subsets" was present in the human liver (82). However, CD49a $\mathrm{a}^{+} \mathrm{NK}$ cells in the human liver may be present in variable quantities.

Cytometry by time of flight can be used to discover and define unique cell populations even if a specific marker for a given subset is not used. By using CyTOF and humanized mice, Yokoyama and colleagues showed that $\mathrm{CD} 49 \mathrm{e}^{-}$is a characteristic marker of trNK cells (25). In addition, several research teams have also reported that the trNK cells observed in the uterus, kidney (83), and salivary glands $(84,85)$ are different from cNK cells in terms of origin, development, and function using “-omics” analysis. Thanks to progress in two-photon microscopy, the discovery of 
extramedullary hematopoiesis in the liver, spleen (86), and even lungs (87) has better defined the origin of trNK cells.

Thanks to "-omics" analysis, the emergence of NK cell diversity based on tissue specificity or the production of different cytokines and the recently identified ILCs have led to a new nomenclature that assigns cNK cells into ILC1s $(88,89)$. Current studies on ILCs are dependent mainly on a mouse model, and technical limitations (e.g., multicolor FCM requires at least eight fluorescence channels; spectral overlap) have hampered adequate characterization of human ILCs. CyTOF provided considerable help to Simoni and colleagues in profiling ILCs from human tissues. Surprisingly, they showed that ILC1s (gating strategy: CD $45^{+}$ $\mathrm{Lin}^{-} \mathrm{CD} 94^{-} \mathrm{CD} 127^{+} \mathrm{CRTH}^{-}{ }^{-}$- $\mathrm{Kit}^{-} \mathrm{NKp} 44^{-}$) were undetectable in human tissues, and an intraepithelial ILC1-like population not restricted to mucosal tissues and which displayed similarity to NK cells was found (90). Bernink et al. showed that NK cells could be distinguished from ILC1s because NK cells highly expressed EOMES, perforin, and granzyme B along with a lack of cell surface expression of CD127 and CD49a (91). A more interesting finding was that NK cells and ILC1s had more closely overlapping gene expression on phenotypes and functional programs (15). Although NK cells have similar functions to ILCs, they may be derived from distinct progenitors and have different requirements for EOMES and T-bet (92). Those studies seem to suggest that these two cell types represent only a subset of the broad NK lineage (93).

The emerging knowledge of the diversity of ILC2s and ILC3s is important $(90,93)$. The diversity of ILCs is a very complicated and confusing problem. A more optimized "multi-omics" analysis uncovered the veil of the diversity of NK cells and allowed us to better understand how cell diversity affects their functions in different tissues in physiologic and pathologic conditions (Figure 2).

\section{"OMICS" ANALYSIS WILL ACCELERATE RESEARCH INTO NK CELLS AND START A NEW CHAPTER IN IMMUNOTHERAPY}

Natural killer cells spontaneously kill cells that are deemed to be "dangerous" to the host, including tumor cells (1) and viruses (94). NK cells have been valuable for fighting against cancer, and researchers are now close to a big breakthrough: NK cells may be able to identify and rapidly kill tumor cells without damaging healthy cells or risking the "storm" of pro-inflammatory cytokines caused by activated $\mathrm{T}$ cells (6).

Recently, due to the wider applications of "-omics" analysis, including scRNA-seq and CyTOF, the classifications and descriptions of NK cell subsets have reached a new level (95). Using CyTOF combined with analyses rooted in epidemiology and population genetics, it not only showed that haplotypes with $-21 \mathrm{M}$ HLA-B rarely encode the KIR ligands Bw4+HLA-B and C2 ${ }^{+}$HLA-C KIR but also showed that stepwise addition of each KIR ligand associated with NK cells helped to "educate" and recognize the responses of CD94:NKG2A and HLA-E $(96,97)$. In fact, those findings suggest new ways to dissect the numerous clinical associations with HLA class-I molecules and are important for the clinical application of NK cells (98). Romee and colleagues investigated the potential of memory-like NK cells in cancer therapy. Through CyTOF, SPADE analysis was used in memory-like NK cells pre-activated by IL-12, IL-15, and IL-18. Results showed that these cells were effective against leukemia targets regardless of KIR-KIR ligand interactions (48). In a study by Miller and colleagues in 2005, this new treatment strategy using pre-activation elicited greater progress than direct transfer of NK cells in inducing the remission of AML (99). As an "offthe-shelf" therapy, on 20 March 2017, the US Food and Drug Administration granted a designation of "orphan drug" to the NantKwest Company for activated NK cell therapy for patients diagnosed with malignant Merkel cell carcinoma. We believe that massive "-omics" data will provide more information to immunologists for developing more accurate and effective NK cell therapy for tumor immunotherapy.

"Omics" analysis is also widely used in antiviral studies using NK cells. Memory-like NK cells have been induced in viral-infected mice (100-102), but the formation mechanism of the pool of memory-like NK cells is not clear. Results of transcriptome and DNA methylation analyses have shown that the formation and maintenance of memory-like NK cells is dependent on the epigenetic changes associated with functional changes (103) and antibody-dependent expansion (104). Moreover, there is insufficient evidence for a correlation between the diversity and the function of NK cells. CyTOF has been used to assess changes in NK cell diversity during human immunodeficiency virus (HIV) infection. Results showed that an increase in NK cell diversity could reduce the ability of expansion and degranulation though promotion of the secretion of cytokines, which resulted in an increased risk of HIV infection (24). Recently, Aguilar et al. identified the viral ligand $\mathrm{m} 12$ for NK1.1 (105) receptors through protein structure-related big data analysis (106). That study has elicited considerable progress in the study of NK cells and has important implications for immunotherapy.

In addition, human cytomegalovirus (HCMV) infection has been shown to be related to some autoimmune diseases (ADs) (107-109) and regulatory NK cells (110-114). An HCMVinduced autoantibody was identified from $\mathrm{AD}$ patients using phage display technology and provided a clear intrinsic connection between reduced numbers of CD56 $6^{\text {bright }} \mathrm{NK}$ cells caused by autoantibodies and AD (115). In addition, during a successful pregnancy, NK cells act as crucial regulatory cells, producing IFN$\gamma$ to suppress Th17-mediated inflammation at the maternal-fetal interface (116). However, the regulation of NK cells has backfired in insulin resistance; experimental data show that stimulated NK cells are linked to obesity-induced adipose stress and lead to increased numbers of pro-inflammatory macrophages and exacerbate insulin resistance $(117,118)$. Irrespective of their use in the treatment of tumors, viral infections, or ADs, NK cells will usher in breakthroughs due to advancements in "-omics" technologies (Figure 2).

\section{CONCLUDING REMARKS}

Natural killer cells are more complicated than originally thought. Due to technical limitations, for a long time, the 
study of NK cells lagged behind those of T cells and B cells. Reviewing the timeline of studies of NK cells, breakthroughs have been in parallel with advances in "-omics" technology. Such advances have not only been translated into new powerful tools but have also rejuvenated research into NK cells. "Omics" technology can provide an overwhelming amount of information in one experiment. Massive amounts of information can give immunologists richer clues and more ample data to better answer questions that remain regarding the biology of NK cells and further enhance understanding of NK cells (Figure 2). Moreover, “-omics" technology is a golden opportunity to accelerate the process of exploring the basic research of NK cells and developing NK cell-mediated immunotherapy to combat various diseases.

\section{REFERENCES}

1. Kiessling R, Klein E, Wigzell H. "Natural" killer cells in the mouse. I. Cytotoxic cells with specificity for mouse Moloney leukemia cells. Specificity and distribution according to genotype. Eur J Immunol (1975) 5(2):112-7. doi:10.1002/eji.1830050208

2. Herberman RR, Ortaldo JR, Bonnard GD. Augmentation by interferon of human natural and antibody-dependent cell-mediated cytotoxicity. Nature (1979) 277(5693):221-3. doi:10.1038/277221a0

3. Ortaldo JR, Bonnard GD, Kind PD, Herberman RB. Cytotoxicity by cultured human lymphocytes: characteristics of effector cells and specificity of cytotoxicity. J Immunol (1979) 122(4):1489-94.

4. Lanier LL, Phillips JH, Hackett J Jr, Tutt M, Kumar V. Natural killer cells: definition of a cell type rather than a function. J Immunol (1986) 137(9):2735-9.

5. Janeway CA. Natural killer cells: a primitive immune system. Nature (1989) 341(6238):108. doi:10.1038/341108a0

6. Guillerey C, Huntington ND, Smyth MJ. Targeting natural killer cells in cancer immunotherapy. Nat Immunol (2016) 17(9):1025-36. doi:10.1038/ ni. 3518

7. Lander ES, Linton LM, Birren B, Nusbaum C, Zody MC, Baldwin J, et al. Initial sequencing and analysis of the human genome. Nature (2001) 409(6822):860-921. doi:10.1038/35057062

8. Venter JC, Adams MD, Myers EW, Li PW, Mural RJ, Sutton GG, et al. The sequence of the human genome. Science (2001) 291(5507):1304-51. doi:10.1126/science. 1058040

9. Waterston RH, Lindblad-Toh K, Birney E, Rogers J, Abril JF, Agarwal P, et al. Initial sequencing and comparative analysis of the mouse genome. Nature (2002) 420(6915):520-62. doi:10.1038/nature01262

10. Lipshutz RJ, Morris D, Chee M, Hubbell E, Kozal MJ, Shah N, et al. Using oligonucleotide probe arrays to access genetic diversity. Biotechniques (1995) 19(3):442-7.

11. McHugh TM. Flow microsphere immunoassay for the quantitative and simultaneous detection of multiple soluble analytes. Methods Cell Biol (1994) 42(Pt B):575-95. doi:10.1016/S0091-679X(08)61096-1

12. Schena M, Shalon D, Davis RW, Brown PO. Quantitative monitoring of gene expression patterns with a complementary DNA microarray. Science (1995) 270(5235):467-70. doi:10.1126/science.270.5235.467

13. Beaulieu AM, Zawislak CL, Nakayama T, Sun JC. The transcription factor Zbtb32 controls the proliferative burst of virus-specific natural killer cells responding to infection. Nat Immunol (2014) 15(6):546-53. doi:10.1038/ ni. 2876

14. Wang F, Zhou Y, Fu B, Wu Y, Zhang R, Sun R, et al. Molecular signatures and transcriptional regulatory networks of human immature decidual NK and mature peripheral NK cells. Eur J Immunol (2014) 44(9):2771-84. doi:10.1002/eji.201344183

15. Robinette ML, Fuchs A, Cortez VS, Lee JS, Wang Y, Durum SK, et al. Transcriptional programs define molecular characteristics of innate lymphoid cell classes and subsets. Nat Immunol (2015) 16(3):306-17. doi:10.1038/ni.3094

\section{AUTHOR CONTRIBUTIONS}

YZ collated data and wrote the review. XX collected data from online databases. ZT and HW conceived and edited the review.

\section{ACKNOWLEDGMENTS}

This work was supported by the key project of the National Natural Science Foundation of China (\#91442202, 81330071).

\section{SUPPLEMENTARY MATERIAL}

The Supplementary Material for this article can be found online at http://journal.frontiersin.org/article/10.3389/fimmu. 2017.01095/full\#supplementary-material.

16. Ni F, Sun R, Fu B, Wang F, Guo C, Tian Z, et al. IGF-1 promotes the development and cytotoxic activity of human NK cells. Nat Commun (2013) 4:1479. doi: $10.1038 /$ ncomms 2484

17. Ni F, Guo C, Sun R, Fu B, Yang Y, Wu L, et al. MicroRNA transcriptomes of distinct human NK cell populations identify miR-362-5p as an essential regulator of NK cell function. Sci Rep (2015) 5:9993. doi:10.1038/srep09993

18. Zhang R, Ni F, Fu B, Wu Y, Sun R, Tian Z, et al. A long noncoding RNA positively regulates CD56 in human natural killer cells. Oncotarget (2016) 7(45):72546-58. doi:10.18632/oncotarget.12466

19. Sojka DK, Plougastel-Douglas B, Yang L, Pak-Wittel MA, Artyomov MN, Ivanova $\mathrm{Y}$, et al. Tissue-resident natural killer (NK) cells are cell lineages distinct from thymic and conventional splenic NK cells. Elife (2014) 3:e01659. doi:10.7554/eLife.01659

20. Bjorklund AK, Forkel M, Picelli S, Konya V, Theorell J, Friberg D, et al. The heterogeneity of human $\mathrm{CD} 127(+)$ innate lymphoid cells revealed by single-cell RNA sequencing. Nat Immunol (2016) 17(4):451-60. doi:10.1038/ ni.3368

21. Yu Y, Tsang JC, Wang C, Clare S, Wang J, Chen X, et al. Single-cell RNA-seq identifies a PD-1hi ILC progenitor and defines its development pathway. Nature (2016) 539(7627):102-6. doi:10.1038/nature20105

22. Fehniger TA, Wylie T, Germino E, Leong JW, Magrini VJ, Koul S, et al. Next-generation sequencing identifies the natural killer cell microRNA transcriptome. Genome Res (2010) 20(11):1590-604. doi:10.1101/gr.107995.110

23. Shih HY, Sciume G, Mikami Y, Guo L, Sun HW, Brooks SR, et al. Developmental acquisition of regulomes underlies innate lymphoid cell functionality. Cell (2016) 165(5):1120-33. doi:10.1016/j.cell.2016.04.029

24. Strauss-Albee DM, Fukuyama J, Liang EC, Yao Y, Jarrell JA, Drake AL, et al. Human NK cell repertoire diversity reflects immune experience and correlates with viral susceptibility. Sci Transl Med (2015) 7(297):297ra115. doi:10.1126/scitranslmed.aac5722

25. Yeang HX, Piersma SJ, Lin Y, Yang L, Malkova ON, Miner C, et al. Cutting edge: human CD49e- NK cells are tissue resident in the liver. J Immunol (2017) 198(4):1417-22. doi:10.4049/jimmunol.1601818

26. Rieckmann JC, Geiger R, Hornburg D, Wolf T, Kveler K, Jarrossay D, et al. Social network architecture of human immune cells unveiled by quantitative proteomics. Nat Immunol (2017) 18(5):583-93. doi:10.1038/ni.3693

27. Chen S, Yang M, Du J, Li D, Li Z, Cai C, et al. The self-specific activation receptor SLAM family is critical for NK cell education. Immunity (2016) 45(2):292-304. doi:10.1016/j.immuni.2016.07.013

28. Heng TS, Painter MW. The immunological genome project: networks of gene expression in immune cells. Nat Immunol (2008) 9(10):1091-4. doi:10.1038/ ni1008-1091

29. Mortazavi A, Williams BA, McCue K, Schaeffer L, Wold B. Mapping and quantifying mammalian transcriptomes by RNA-Seq. Nat Methods (2008) 5(7):621-8. doi:10.1038/nmeth.1226

30. Sultan M, Schulz MH, Richard H, Magen A, Klingenhoff A, Scherf M, et al. A global view of gene activity and alternative splicing by deep sequencing of the human transcriptome. Science (2008) 321(5891):956-60. doi:10.1126/ science. 1160342 
31. Blow N. Transcriptomics: the digital generation. Nature (2009) 458(7235):239-42. doi:10.1038/458239a

32. Peng H, Jiang X, Chen Y, Sojka DK, Wei H, Gao X, et al. Liver-resident NK cells confer adaptive immunity in skin-contact inflammation. JClin Invest (2013) 123(4):1444-56. doi:10.1172/jci66381

33. Pennisi E. The biology of genomes. Single-cell sequencing tackles basic and biomedical questions. Science (2012) 336(6084):976-7. doi:10.1126/ science.336.6084.976

34. Tang F, Barbacioru C, Wang Y, Nordman E, Lee C, Xu N, et al. mRNA-Seq whole-transcriptome analysis of a single cell. Nat Methods (2009) 6(5):377-82. doi:10.1038/nmeth.1315

35. Spits H, Artis D, Colonna M, Diefenbach A, Di Santo JP, Eberl G, et al. Innate lymphoid cells - a proposal for uniform nomenclature. Nat Rev Immunol (2013) 13(2):145-9. doi:10.1038/nri3365

36. Artis D, Spits H. The biology of innate lymphoid cells. Nature (2015) 517(7534):293-301. doi:10.1038/nature14189

37. Eberl G, Colonna M, Di Santo JP, McKenzie AN. Innate lymphoid cells. Innate lymphoid cells: a new paradigm in immunology. Science (2015) 348(6237):aaa6566. doi:10.1126/science.aaa6566

38. Barski A, Cuddapah S, Cui K, Roh TY, Schones DE, Wang Z, et al. Highresolution profiling of histone methylations in the human genome. Cell (2007) 129(4):823-37. doi:10.1016/j.cell.2007.05.009

39. Buenrostro JD, Giresi PG, Zaba LC, Chang HY, Greenleaf WJ. Transposition of native chromatin for fast and sensitive epigenomic profiling of open chromatin, DNA-binding proteins and nucleosome position. Nat Methods (2013) 10(12):1213-8. doi:10.1038/nmeth.2688

40. Buenrostro JD, Wu B, Chang HY, Greenleaf WJ. ATAC-seq: a method for assaying chromatin accessibility genome-wide. Curr Protoc Mol Biol (2015) 109:21.29.1-9. doi:10.1002/0471142727.mb2129s109

41. Williams AF, Galfre G, Milstein C. Analysis of cell surfaces by xenogeneic myeloma-hybrid antibodies: differentiation antigens of rat lymphocytes. Cell (1977) 12(3):663-73. doi:10.1016/0092-8674(77)90266-5

42. Bendall SC, Simonds EF, Qiu P, Amir el AD, Krutzik PO, Finck R, et al. Single-cell mass cytometry of differential immune and drug responses across a human hematopoietic continuum. Science (2011) 332(6030):687-96. doi:10.1126/science.1198704

43. Spitzer MH, Nolan GP. Mass cytometry: single cells, many features. Cell (2016) 165(4):780-91. doi:10.1016/j.cell.2016.04.019

44. Newell EW, Sigal N, Bendall SC, Nolan GP, Davis MM. Cytometry by timeof-flight shows combinatorial cytokine expression and virus-specific cell niches within a continuum of CD8+ T cell phenotypes. Immunity (2012) 36(1):142-52. doi:10.1016/j.immuni.2012.01.002

45. Horowitz A, Strauss-Albee DM, Leipold M, Kubo J, Nemat-Gorgani N, Dogan OC, et al. Genetic and environmental determinants of human NK cell diversity revealed by mass cytometry. Sci Transl Med (2013) 5(208):208ra145. doi:10.1126/scitranslmed.3006702

46. Leavy O. Natural killer cells: a virtual pick and mix. Nat Rev Immunol (2013) 13(12):844-5. doi:10.1038/nri3566

47. Horowitz A, Guethlein LA, Nemat-Gorgani N, Norman PJ, Cooley S, Miller JS, et al. Regulation of adaptive NK cells and CD8 T cells by HLA-C correlates with allogeneic hematopoietic cell transplantation and with cytomegalovirus reactivation. J Immunol (2015) 195(9):4524-36. doi:10.4049/jimmunol.1401990

48. Romee R, Rosario M, Berrien-Elliott MM, Wagner JA, Jewell BA, Schappe T, et al. Cytokine-induced memory-like natural killer cells exhibit enhanced responses against myeloid leukemia. Sci Transl Med (2016) 8(357):357ra123. doi:10.1126/scitranslmed.aaf2341

49. Peng J, Elias JE, Thoreen CC, Licklider LJ, Gygi SP. Evaluation of multidimensional chromatography coupled with tandem mass spectrometry (LC/ LC-MS/MS) for large-scale protein analysis: the yeast proteome. J Proteome Res (2003) 2(1):43-50. doi:10.1021/pr025556v

50. Cong L, Ran FA, Cox D, Lin S, Barretto R, Habib N, et al. Multiplex genome engineering using CRISPR/Cas systems. Science (2013) 339(6121):819-23. doi: $10.1126 /$ science. 1231143

51. Yang $\mathrm{H}$, Wang $\mathrm{H}$, Jaenisch R. Generating genetically modified mice using CRISPR/Cas-mediated genome engineering. Nat Protoc (2014) 9(8):1956-68. doi:10.1038/nprot.2014.134

52. Rong H, Shi Y. Disruption of key GTPase regulators of endocytic recycling compartment does not interfere with soluble antigen crosspresentation in dendritic cells. Cell Mol Immunol (2016) 13(4):554-6. doi:10.1038/cmi.2015.17
53. Yang L, Xu LZ, Liu ZQ, Yang G, Geng XR, Mo LH, et al. Interleukin-13 interferes with activation-induced t-cell apoptosis by repressing p 53 expression. Cell Mol Immunol (2016) 13(5):669-77. doi:10.1038/cmi.2015.50

54. Sun C, Sun H, Zhang C, Tian Z. NK cell receptor imbalance and NK cell dysfunction in HBV infection and hepatocellular carcinoma. Cell Mol Immunol (2015) 12(3):292-302. doi:10.1038/cmi.2014.91

55. Vivier E, van de Pavert SA, Cooper MD, Belz GT. The evolution of innate lymphoid cells. Nat Immunol (2016) 17(7):790-4. doi:10.1038/ni.3459

56. Yoder JA, Litman GW. The phylogenetic origins of natural killer receptors and recognition: relationships, possibilities, and realities. Immunogenetics (2011) 63(3):123-41. doi:10.1007/s00251-010-0506-4

57. Straub C, Neulen ML, Sperling B, Windau K, Zechmann M, Jansen CA, et al. Chicken NK cell receptors. Dev Comp Immunol (2013) 41(3):324-33. doi:10.1016/j.dci.2013.03.013

58. Carrillo-Bustamante P, Kesmir C, de Boer RJ. The evolution of natural killer cell receptors. Immunogenetics (2016) 68(1):3-18. doi:10.1007/ s00251-015-0869-7

59. Lavin Y, Kobayashi S, Leader A, Amir ED, Elefant N, Bigenwald C, et al. Innate immune landscape in early lung adenocarcinoma by paired single-cell analyses. Cell (2017) 169(4):750-65.e17. doi:10.1016/j.cell.2017.04.014

60. Gascoyne DM, Long E, Veiga-Fernandes H, de Boer J, Williams O, Seddon B, et al. The basic leucine zipper transcription factor E4BP4 is essential for natural killer cell development. Nat Immunol (2009) 10(10):1118-24. doi:10.1038/ni.1787

61. Kamizono S, Duncan GS, Seidel MG, Morimoto A, Hamada K, Grosveld G, et al. Nfil3/E4bp4 is required for the development and maturation of NK cells in vivo. J Exp Med (2009) 206(13):2977-86. doi:10.1084/jem.20092176

62. Deng Y, Kerdiles Y, Chu J, Yuan S, Wang Y, Chen X, et al. Transcription factor Foxol is a negative regulator of natural killer cell maturation and function. Immunity (2015) 42(3):457-70. doi:10.1016/j.immuni.2015.02.006

63. Carson WE, Giri JG, Lindemann MJ, Linett ML, Ahdieh M, Paxton R, et al. Interleukin (IL) 15 is a novel cytokine that activates human natural killer cells via components of the IL-2 receptor. J Exp Med (1994) 180(4):1395-403. doi:10.1084/jem.180.4.1395

64. Male V, Nisoli I, Kostrzewski T, Allan DS, Carlyle JR, Lord GM, et al. The transcription factor E4bp4/Nfil3 controls commitment to the NK lineage and directly regulates Eomes and Id2 expression. J Exp Med (2014) 211(4):635-42. doi:10.1084/jem.20132398

65. Yang M, Chen S, Du J, He J, Wang Y, Li Z, et al. NK cell development requires Tsc1-dependent negative regulation of IL-15-triggered mTORC1 activation. Nat Commun (2016) 7:12730. doi:10.1038/ncomms12730

66. Simonetta F, Pradier A, Roosnek E. T-bet and eomesodermin in NK cell development, maturation, and function. Front Immunol (2016) 7:241. doi:10.3389/fimmu.2016.00241

67. Szabo SJ, Kim ST, Costa GL, Zhang X, Fathman CG, Glimcher LH. A novel transcription factor, T-bet, directs Th1 lineage commitment. Cell (2000) 100(6):655-69. doi:10.1016/S0092-8674(00)80702-3

68. Townsend MJ, Weinmann AS, Matsuda JL, Salomon R, Farnham PJ, Biron CA, et al. T-bet regulates the terminal maturation and homeostasis of NK and Valpha14i NKT cells. Immunity (2004) 20(4):477-94. doi:10.1016/ S1074-7613(04)00076-7

69. Jenne CN, Enders A, Rivera R, Watson SR, Bankovich AJ, Pereira JP, et al. T-bet-dependent S1P5 expression in NK cells promotes egress from lymph nodes and bone marrow. J Exp Med (2009) 206(11):2469-81. doi:10.1084/ jem. 20090525

70. Kallies A, Carotta S, Huntington ND, Bernard NJ, Tarlinton DM, Smyth MJ, et al. A role for Blimp1 in the transcriptional network controlling natural killer cell maturation. Blood (2011) 117(6):1869-79. doi:10.1182/ blood-2010-08-303123

71. Tang PM, Zhou S, Meng XM, Wang QM, Li CJ, Lian GY, et al. Smad3 promotes cancer progression by inhibiting E4BP4-mediated NK cell development. Nat Commun (2017) 8:14677. doi:10.1038/ncomms14677

72. Sunwoo JB, Kim S, Yang L, Naik T, Higuchi DA, Rubenstein JL, et al. Distalless homeobox transcription factors regulate development and maturation of natural killer cells. Proc Natl Acad Sci U S A (2008) 105(31):10877-82. doi:10.1073/pnas. 0805205105

73. Liu S, Chen L, Zeng Y, Si L, Guo X, Zhou J, et al. Suppressed expression of miR-378 targeting gzmb in NK cells is required to control dengue virus infection. Cell Mol Immunol (2016) 13(5):700-8. doi:10.1038/cmi.2015.52 
74. Goh W, Huntington ND. Regulation of murine natural killer cell development. Front Immunol (2017) 8:130. doi:10.3389/fimmu.2017.00130

75. Sojka DK, Tian Z, Yokoyama WM. Tissue-resident natural killer cells and their potential diversity. Semin Immunol (2014) 26(2):127-31. doi:10.1016/j. smim.2014.01.010

76. Bjorkstrom NK, Ljunggren HG, Michaelsson J. Emerging insights into natural killer cells in human peripheral tissues. Nat Rev Immunol (2016) 16(5):310-20. doi:10.1038/nri.2016.34

77. Hulett HR, Bonner WA, Barrett J, Herzenberg LA. Cell sorting: automated separation of mammalian cells as a function of intracellular fluorescence. Science (1969) 166(3906):747-9. doi:10.1126/science.166.3906.747

78. Peng $\mathrm{H}$, Tian $\mathrm{Z}$. Re-examining the origin and function of liver-resident NK cells. Trends Immunol (2015) 36(5):293-9. doi:10.1016/j.it.2015.03.006

79. Gao B. Basic liver immunology. Cell Mol Immunol (2016) 13(3):265-6. doi:10.1038/cmi.2016.09

80. Peng H, Wisse E, Tian Z. Liver natural killer cells: subsets and roles in liver immunity. Cell Mol Immunol (2016) 13(3):328-36. doi:10.1038/cmi.2015.96

81. Robinson MW, Harmon C, O'Farrelly C. Liver immunology and its role in inflammation and homeostasis. Cell Mol Immunol (2016) 13(3):267-76. doi:10.1038/cmi.2016.3

82. Marquardt N, Beziat V, Nystrom S, Hengst J, Ivarsson MA, Kekalainen E, et al. Cutting edge: identification and characterization of human intrahepatic CD49a+ NK cells. J Immunol (2015) 194(6):2467-71. doi:10.4049/ jimmunol.1402756

83. Victorino F, Sojka DK, Brodsky KS, McNamee EN, Masterson JC, Homann D, et al. Tissue-resident NK cells mediate ischemic kidney injury and are not depleted by anti-asialo-GM1 antibody. JImmunol (2015) 195(10):4973-85. doi:10.4049/jimmunol.1500651

84. Cortez VS, Fuchs A, Cella M, Gilfillan S, Colonna M. Cutting edge: salivary gland NK cells develop independently of Nfil3 in steady-state. J Immunol (2014) 192(10):4487-91. doi:10.4049/jimmunol.1303469

85. Cortez VS, Cervantes-Barragan L, Robinette ML, Bando JK, Wang Y, Geiger TL, et al. Transforming growth factor-beta signaling guides the differentiation of innate lymphoid cells in salivary glands. Immunity (2016) 44(5):1127-39. doi:10.1016/j.immuni.2016.03.007

86. Inra CN, Zhou BO, Acar M, Murphy MM, Richardson J, Zhao Z, et al. A perisinusoidal niche for extramedullary haematopoiesis in the spleen. Nature (2015) 527(7579):466-71. doi:10.1038/nature15530

87. Lefrancais E, Ortiz-Munoz G, Caudrillier A, Mallavia B, Liu F, Sayah DM, et al. The lung is a site of platelet biogenesis and a reservoir for haematopoietic progenitors. Nature (2017) 544(7648):105-9. doi:10.1038/nature21706

88. Liu J, Cao X. Cellular and molecular regulation of innate inflammatory responses. Cell Mol Immunol (2016) 13(6):711-21. doi:10.1038/cmi.2016.58

89. Spits H, Bernink JH, Lanier L. NK cells and type 1 innate lymphoid cells: partners in host defense. Nat Immunol (2016) 17(7):758-64. doi:10.1038/ni.3482

90. Simoni Y, Fehlings M, Kloverpris HN, McGovern N, Koo SL, Loh CY, et al. Human innate lymphoid cell subsets possess tissue-type based heterogeneity in phenotype and frequency. Immunity (2017) 46(1):148-61. doi:10.1016/j. immuni.2016.11.005

91. Bernink JH, Peters CP, Munneke M, te Velde AA, Meijer SL, Weijer K, et al. Human type 1 innate lymphoid cells accumulate in inflamed mucosal tissues. Nat Immunol (2013) 14(3):221-9. doi:10.1038/ni.2534

92. Tang L, Peng H, Zhou J, Chen Y, Wei H, Sun R, et al. Differential phenotypic and functional properties of liver-resident NK cells and mucosal ILC1s. J Autoimmun (2016) 67:29-35. doi:10.1016/j.jaut.2015.09.004

93. Ealey KN, Koyasu S. How many subsets of innate lymphoid cells do we need? Immunity (2017) 46(1):10-3. doi:10.1016/j.immuni.2016.12.018

94. Biron CA, Byron KS, Sullivan JL. Severe herpesvirus infections in an adolescent without natural killer cells. N Engl J Med (1989) 320(26):1731-5. doi:10.1056/nejm198906293202605

95. Keener AB. Natural killers: cataloging immune cells for immunotherapy. Nat Med (2015) 21(3):207-8. doi:10.1038/nm0315-207

96. Horowitz A, Djaoud Z, Nemat-Gorgani N, Blokhuis J, Hilton HG, Béziat V, et al. Class I HLA haplotypes form two schools that educate NK cells in different ways. Sci Immunol (2016) 1(3):eaag1672. doi:10.1126/sciimmunol.aag1672

97. He Y, Tian Z. NK cell education via nonclassical MHC and non-MHC ligands. Cell Mol Immunol (2017) 14(4):321-30. doi:10.1038/cmi.2016.26

98. Cheng M, Chen Y, Xiao W, Sun R, Tian Z. NK cell-based immunotherapy for malignant diseases. Cell Mol Immunol (2013) 10(3):230-52. doi:10.1038/ cmi.2013.10
99. Miller JS, Soignier Y, Panoskaltsis-Mortari A, McNearney SA, Yun GH, Fautsch SK, et al. Successful adoptive transfer and in vivo expansion of human haploidentical NK cells in patients with cancer. Blood (2005) 105(8):3051-7. doi:10.1182/blood-2004-07-2974

100. Sun JC, Beilke JN, Lanier LL. Adaptive immune features of natural killer cells. Nature (2009) 457(7229):557-61. doi:10.1038/nature07665

101. O'Sullivan TE, Sun JC, Lanier LL. Natural killer cell memory. Immunity (2015) 43(4):634-45. doi:10.1016/j.immuni.2015.09.013

102. Cerwenka A, Lanier LL. Natural killer cell memory in infection, inflammation and cancer. Nat Rev Immunol (2016) 16(2):112-23. doi:10.1038/nri.2015.9

103. Schlums H, Cichocki F, Tesi B, Theorell J, Beziat V, Holmes TD, et al. Cytomegalovirus infection drives adaptive epigenetic diversification of NK cells with altered signaling and effector function. Immunity (2015) 42(3):443-56. doi:10.1016/j.immuni.2015.02.008

104. Lee J, Zhang T, Hwang I, Kim A, Nitschke L, Kim M, et al. Epigenetic modification and antibody-dependent expansion of memory-like NK cells in human cytomegalovirus-infected individuals. Immunity (2015) 42(3):431-42. doi:10.1016/j.immuni.2015.02.013

105. Koo GC, Peppard JR. Establishment of monoclonal anti-Nk-1.1 antibody. Hybridoma (1984) 3(3):301-3. doi:10.1089/hyb.1984.3.301

106. Aguilar OA, Berry R, Rahim MM, Reichel JJ, Popovic B, Tanaka M, et al. A viral immunoevasin controls innate immunity by targeting the prototypical natural killer cell receptor family. Cell (2017) 169(1):58-71.e14. doi:10.1016/j. cell.2017.03.002

107. Lunardi C, Bason C, Navone R, Millo E, Damonte G, Corrocher R, et al. Systemic sclerosis immunoglobulin $\mathrm{G}$ autoantibodies bind the human cytomegalovirus late protein UL94 and induce apoptosis in human endothelial cells. Nat Med (2000) 6(10):1183-6. doi:10.1038/80533

108. Soderberg-Naucler C. Autoimmunity induced by human cytomegalovirus in patients with systemic lupus erythematosus. Arthritis Res Ther (2012) 14(1):101. doi:10.1186/ar3525

109. Halenius A, Hengel H. Human cytomegalovirus and autoimmune disease. Biomed Res Int (2014) 2014:472978. doi:10.1155/2014/472978

110. Vivier E, Ugolini S. Regulatory natural killer cells: new players in the IL-10 anti-inflammatory response. Cell Host Microbe (2009) 6(6):493-5. doi:10.1016/j.chom.2009.12.001

111. Tian Z, Gershwin ME, Zhang C. Regulatory NK cells in autoimmune disease. J Autoimmun (2012) 39(3):206-15. doi:10.1016/j.jaut.2012.05.006

112. Jonjic S. CMV immunology. Cell Mol Immunol (2015) 12(2):125-7. doi:10.1038/cmi.2014.132

113. Gross CC, Schulte-Mecklenbeck A, Wiendl H, Marcenaro E, Kerlero de Rosbo N, Uccelli A, et al. Regulatory functions of natural killer cells in multiple sclerosis. Front Immunol (2016) 7:606. doi:10.3389/fimmu.2016.00606

114. Zhang C, Tian Z. NK cell subsets in autoimmune diseases. J Autoimmun (2017) 83:22-30. doi:10.1016/j.jaut.2017.02.005

115. Liu Y, Mu R, Gao YP, Dong J, Zhu L, Ma Y, et al. A cytomegalovirus peptide-specific antibody alters natural killer cell homeostasis and is shared in several autoimmune diseases. Cell Host Microbe (2016) 19(3):400-8. doi:10.1016/j.chom.2016.02.005

116. Fu B, Li X, Sun R, Tong X, Ling B, Tian Z, et al. Natural killer cells promote immune tolerance by regulating inflammatory TH17 cells at the human maternal-fetal interface. Proc Natl Acad Sci U S A (2013) 110(3):E231-40. doi:10.1073/pnas.1206322110

117. Wensveen FM, Jelencic V, Valentic S, Sestan M, Wensveen TT, Theurich S, et al. NK cells link obesity-induced adipose stress to inflammation and insulin resistance. Nat Immunol (2015) 16(4):376-85. doi:10.1038/ni.3120

118. Lee BC, Kim MS, Pae M, Yamamoto Y, Eberle D, Shimada T, et al. Adipose natural killer cells regulate adipose tissue macrophages to promote insulin resistance in obesity. Cell Metab (2016) 23(4):685-98. doi:10.1016/j.cmet.2016.03.002

Conflict of Interest Statement: The authors declare that the research was conducted in the absence of any commercial or financial relationships that could be construed as a potential conflict of interest.

Copyright $\odot 2017$ Zhou, Xu, Tian and Wei. This is an open-access article distributed under the terms of the Creative Commons Attribution License (CC BY). The use, distribution or reproduction in other forums is permitted, provided the original author(s) or licensor are credited and that the original publication in this journal is cited, in accordance with accepted academic practice. No use, distribution or reproduction is permitted which does not comply with these terms. 